\title{
Desarrollo del aprendizaje de la escritura en alumnos y alumnas discapacitados
}

\section{Neus Roca Cortés}

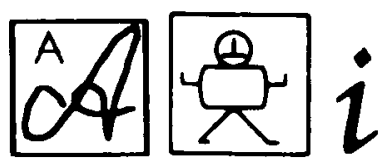

En este artículo se pasa el énfasis de los alumnos con discapacidad desde su limitación a su proceso de construcción y desarrollo, analizando desde abi muchas de las estrtegias de tareas $y$ destrezas y sus efectos, con frecuencia negativos, apoyándose por el contrario en su proceso de construcción y viendo sus errores como mecanismos constructivos en la Zona de Desarrollo Próximo hacia formas progresivamente más elaboradas de escritura. El artículo, aún siguiendo el bilo expositivo de una investigación, ofrece un modelo alternativo claro para el diseño didáctico.

\section{INTRODUCCION}

La lectura, la escritura y en general el dominio del lenguaje escrito constituye uno de los principales objetivos de la enseñanza especial. A su consecución, las enseñantes le dedican una buena parte de los esfuerzos cotidianos, ya que se trata de una de las principales áreas curriculares seleccionadas en la adaptación del currículum a las necesidades educativas individuales y peculiares del alumnado que presenta algún tipo de discapacitación, retraso o alteración.

Algunos enfoques actuales sobre lectura y escritura aportan nuevas perspectivas que prometen ser alentadoras en el campo de la enseñanza-aprendizaje de alumnos con necesidades educativas especiales.

Diversas investigaciones, iniciadas por Ferreiro y colaboradores (1979), enmarcadas en la orientación psicogenética y la piscolingüística postchomskyana, han mostrado diferentes esquemas que la niña y el niño se plantean en el desarrollo del conocimiento de la lectura y la escritura.

Para el trabajo que aquí nos ocupa, cabe destacar un aspecto clave que -a nuestro entender - aportan dichas investigaciones: la interpretación, en un conjunto conceptual coherente y fundamento, de producciones escritas e interpre- 
taciones lectoras no convencionales y de las hipótesis subyacentes en tales comportamientos.

Esta posibilidad de interpretación ha sido uno de los motivos principales por los cuales decidimos usar este enfoque en nuestra práctica psicopedagógica y docente en la educación especial. En el transcurso del largo tiempo de aprendizaje de este tipo de alumnos podríamos considerar realmente abundante la cantidad de escrituras y lecturas «incorrectas» que producen. Incorrectas, en el sentido de que usan inadecuadamente el sistema convencional de notación, no atendiendo a una correcta asociación grafía-sonido. Intuíamos, pues, que esas interpretaciones psicogenéticas del lenguaje escrito aplicadas a la práctica de la educación especial podrían ser una ayuda importante al esfuerzo de profesionales y alumnado.

Recogíamos así una de las conclusiones de E. Ferreiro y M. Gómez-Palacio, en su amplio estudio de Méjico (1982):

«... es legítimo suponer que quienes avanzaron hasta los niveles intermediarios pueden seguir avanzando normalmente, ya que sus compañeros (los que sí llegaron) también pasaron por estas etapas intermediarias y no se detuvieron allí sino que siguieron avanzando.»

El estudio que presentamos forma parte de una experiencia e innovación educativa de aplicación de un enfoque constructivista en la enseñanza de alumnos y alumnas con diversas discapacidades (Roca Cortés, Simó Aguadé, Solsona Roca, González García, Rabasa del Campo, 1991), desarrollado en el ámbito formativo de un grupo espontáneo y estable de profesionales de la enseñanza primaria, pertenecientes al Seminario de Educación Especial de la comarca del Garraf (Barcelona-Cataluña) del Institut de Ciencies de l'Educació de la Universitat de Barcelona.

En este artículo relatamos aquellos aspectos que se refieren al proceso de aprendizaje de la escritura en alumnado con discapacidades y algunas de las implicaciones psicopedagógicas que se derivan que nos obligan a revisar criterios de evaluación y enseñanza al uso. Proponemos cambios en el diagnóstico y en la enseñanza alertando sobre algunas prácticas educativas que empobrecen la educación del alumnado con inconvenientes.

\section{Objetivo}

El objetivo de este estudio ha consistido en la comprobación de si las etapas del proceso de adquisición del sistema alfabético notacional, en muestras amplias de población, descritas por las autoras antes mencionadas, serviría para comprender las escrituras de estos alumnos y alumnas con diversas deficiencias o alteraciones en su escolaridad y si la sucesión de tales etapas serviría también para describir un proceso de adquisición del sistema alfabético en ellos.

\section{El proceso de adquisición de la escritura}

Unos brevísimos apuntes acerca de los puntos claves de las investigaciones anteriormente citadas, permitirán desplegar con más claridad los resultados, conclusiones e implicaciones de nuestro trabajo.

Aunque sometido a la influencia cultural y social, el aprendizaje de la escritura y la lectura puede desarrollarse con cierta independencia de los métodos de enseñanza. Aquello que este enfoque pretende conocer son los conocimien- 
tos sobre el sistema de notación del lenguaje que el niño va desarrollando a lo largo de su evolución. El objeto de conocimiento del niño es, pues, el sistema en sí mismo, concretamente el sistema alfabético (ni silábico ni ideográfico) de notación de nuestras lenguas. En nuestro caso se tratará, pues, del catalán y del castellano.

Teberosky (1988) se expresa así:

«Cuando se estudian las producciones escritas realizadas de manera espontánea, es decir, sin copia, se puede inferir un proceso de desarrollo desde el descubrimiento de las propiedades de los elementos que sirven a la representación escrita hasta el descubrimiento de las reglas de la representación misma.»

En este sentido, cabría considerar que tanto la lectura como la escritura son dos actividades de uso del sistema antes mencionado y que no se identifica la primera con desciframiento ni la segunda con copia de un modelo.

Desde esta perspectiva psicogenética, los criterios de evaluación de las escrituras no se referirán solamente a la escritura alfabética convencional. Serán también tenidos en cuenta los conocimientos que muestra el niño sobre ésta en momentos anteriores al uso del sistema de forma convencional y el tipo de progreso que se va definiendo. La escritura y la lectura alfabéticas serán el final de un largo proceso y no su comienzo.

Desde que el niño y la niña no conocen nada acerca de la escritura hasta que la usan de forma convencional se ha podido categorizar el desempeño en la producción escrita en diferentes etapas (Ferreiro y Teberosky, 1979; Ferreiro y Gómez Palacio, 1982; Tolchinsky y Levin, 1982; Teberosky y Bellés, 1984, etc.).

Presentamos una síntesis en estos niveles (Teberosky, 1989):

a) escrituras presilábicas

a.1) escrituras indiferenciadas. Igual serie de grafías cualquiera que sea el enunciado que el niño se propone escribir. Las grafías pueden ser convencionales, seudoconvencionales o garabatos.

a.2) escrituras diferenciadas: a enunciados significativamente diferentes corresponden series gráficas diferentes. Dos objetos o estímulos diferentes no pueden ser representados de la misma manera. La diferenciación se realiza por variaciones en el repertorio, la cantidad y la posición de las grafías. En estas variaciones suele influir el recuerdo de algún modelo de escritura.

b) escrituras silábicas: la representación gráfica se pone en correspondencia con la sonoridad del enunciado. Esta relación no es la convenionalmente esperada, se trata de una correspondencia en que la silaba es la unidad del análisis sonoro que se hará corresponder a una unidad gráfica, habitualmente una letra (primero vocales y luego consonantes). Paulatinamente, se va adquiriendo una correspondencia de tipo cualitativo en la que determinadas letras y no otras «valen» para determinadas sílabas, usando así las letras con el valor sonoro convencional.

c) escrituras alfabéticas: la correspondencia que se establece entre el enunciado y la producción gráfica sigue a través del análisis sonoro del enunciado pero esta vez la unidad es ya el sonido. La correspondencia es de tipo alfabético (sonido-grafía) y habitualmente se apoya en el valor sonoro convencional.

En el transcurso de este aprendizaje el niño desarrolla unas exigencias que usa para interpretar y escribir. Se trata de la exigencia de una cantidad mínima de grafismos para que algo escrito pueda ser interpretable, y la exigencia de variedad gráfica interna en dos grafismos seguidos para que pueda «decir» algo. Se- 
gún el nivel de desempeño en el que se encuentre resolverá de una forma u otra los conflictos que le provoquen tales exigencias.

\section{DESARROLLO DEL ESTUDIO}

Se ha realizado un seguimiento longitudinal de la escritura durante 2,3 y 4 años, de 2 alumnas y 6 alumnos, de extracción social baja y media-baja.

\section{Condiciones personales de los sujetos}

Se trata, pues, de 8 sujetos, de edades comprendidas entre 5 y 13 años que presentan dificultades para un rendimiento normalizado debido a:

a) transtornos derivados de deficiencias mentales leves (sujetos 1,2 y 3), retrasos de rendimiento tipo límite (sujetos 5 y 6 ), parálisis cerebral con afectaciones muy y menos graves (sujetos 6 y 7); b) no haber estado nunca escolarizado (sujeto 8).

Ninguno de ellos ni ellas presentan afectaciones de tipo afásico y/o sensorial.

En la tabla I se describen para cada sujeto sus condiciones personales y de escolarización, en los siguientes términos:

1. Descripción de la alteración: el tipo de deficiencia o alteración, el grado de afectación, el tipo de dificultades en el lenguaje oral.

2. Datos personales: sexo, edades, siendo la primera aquella en la que se pasó la primera prueba para este estudio, y la segunda, la edad en la que se pasó la última de las pruebas.

3. Datos escolares: aula en la que está escolarizado la mayor parte del horario escolar (A.O. = aula ordinaria, G.R. = grupo reducido de alumnos de educación especial), ayudas especializadas que recibe o ha recibido (E.E. = maestra de educación especial, $L .=$ tratamiento logopédico).

\section{Condiciones de escolarización}

Todos ellos están escolarizados en escuelas públicas ordinarias, y en situación de enseñanza-aprendizaje de la lectoescritura correspondiente a niveles de parvulario y ciclo inicial. Todos ellos son considerados alumnos con necesidades educativas específicas y especiales, sean cuales fueran las enfermedades, los transtornos o circunstancias que haya provocado tal consideración. Se encuentran integrados o en el aula ordinaria (aula común) o en la escuela común.

El método de enseñanza de la lecto-escritura que se aplicaba - tanto en el aula común como en el aula de educación especial- era mayoritariamente un método de tipo fonético (presentación y ejercitación progresiva de cada una de las asociaciones grafía-sonido a partir de las palabras que sólo contengan las letras presentadas hasta el momento y de una correcta pronunciación de los sonidos). Se acompañaba de la estimulación de habilidades supuestamente preparatorias de la madurez para tal aprendizaje (orientación espacial, organización perceptiva, atención, memoria, reproducción de estructuras rítmicas, grafomotricidad, etc.). Estas habilidades preparatorias son recomendadas por numerosos textos de ejercicios, tal como se encuentran en el mercado, para alumnos con dificultades en la lectoescritura, con el objetivo de corregir sus errores e incluso para prevenirlos.

Desde el inicio de nuestro estudio -y solamente en el aula de educación 
TABLA I

Descripción de los sujetos

\begin{tabular}{|c|c|c|c|c|}
\hline Sujeto & Tipo de discapacidad o alteración & Sexo & Edad & Escolarización \\
\hline 1 & $\begin{array}{l}\text { Retraso mental leve (Wais, QI 56), hemi- } \\
\text { paresia, transtornos en la pronunciación y } \\
\text { estructuración del lenguaje. }\end{array}$ & mujer & $7-11$ años & A. O. con ayuda E.E. \\
\hline 2 & $\begin{array}{l}\text { Retraso mental leve (Wais, QI 68), trans- } \\
\text { tornos en la pronunciación del lenguaje. }\end{array}$ & mujer & $7-10$ años & A.O. con ayuda E.E. \\
\hline 3 & $\begin{array}{l}\text { Retraso mental leve (Columbia, Indice 55): } \\
\text { síndrome de down, transtornos en la pro- } \\
\text { nunciación y estructuración del lenguaje. }\end{array}$ & hombre & $6-10$ años & A.O. con ayuda E.E. \\
\hline 4 & $\begin{array}{l}\text { Retraso límite en el desarrollo (McArthy, } \\
\text { CGI 85), transtornos en la pronunciación } \\
\text { del lenguaje. }\end{array}$ & hombre & $5-9$ años & A.O. con ayuda E.E. \\
\hline 5 & $\begin{array}{l}\text { Retraso límite en el desarrollo (McArthy, } \\
\text { CGI 82). }\end{array}$ & hombre & $5-8$ años & A.O. con ayuda E.E. \\
\hline 6 & $\begin{array}{l}\text { Parálisis cerebral, afectación motriz media, } \\
\text { transtornos en la pronunciación y estruc- } \\
\text { turación del lenguaje. }\end{array}$ & hombre & $11-13$ años & G.R. con ayuda L. \\
\hline 7 & $\begin{array}{l}\text { Parálisis cerebral con inmovilidad casi to- } \\
\text { tal. Apenas mueve los ojos y la lengua. Sólo } \\
\text { emite algunos sonidos. }\end{array}$ & hombre & $8-10$ años & G.R. con ayuda L. \\
\hline 8 & $\begin{array}{l}\text { No escolarizado por diversidad cultural, } \\
\text { asiste a la escuela sólo 12 meses, de forma } \\
\text { intermitente. No presenta dificultades en } \\
\text { el lenguaje. }\end{array}$ & hombre & 11 años & A.O. con ayuda E.E. \\
\hline
\end{tabular}

especial - todos ellos pasan paulatinamente a ser enseñados con criterios pedagógicos basados en un enfoque psicolingüístico y constructivista y teniendo en cuenta los sistemas de desarrollo de la escritura propios del niño (siguiendo la propuesta de Teberosky, 1982, 1984, y Teberosky y colaboradoras, 1987, 1989). Entre otros, los principales criterios en que nos basamos son: partir de los conocimientos, hipótesis e informaciones del alumnado, uso de textos escolares (cuento, poesía, etc.) y no escolares (listas de compra, otras listas, carteles, logotipos, comics, notas, etc.) en sus funciones correspondientes y con sus aportaciones habituales, y facilitar al alumnado la ejercitación y contraste de sus propias hipótesis (actividades de escritura de nombres y textos sin modelo desde los inicios de su aprendizaje). Tanto la lectura como la escritura han sido objeto de enseñanza, y además de forma simultánea como usos complementarios que son. En el aula común siguieron en un principio con el método y enfoque antes mencionado. Al cabo de unos años del inicio de este trabajo en el aula de educación especial, algunas aulas ordinarias - en ciclos - iniciaron la aplicación de un enfoque constructivista.

\section{La prueba de evaluación}

En cada curso escolar suelen realizarse tres pruebas de escritura, generalmente al principio del primer trimestre, a principios del segundo trimestre y al final del curso. Los datos para este estudio han sido recogidos de esta tarea de evaluación continuada que realizaban las maestras y la asesora piscopedagógica. 
La prueba de escritura trata de un dictado de cuatro palabras (con diferentes números de sîlabas: mono, bi, tri y letrasílabas) y una frase, en el marco de una entrevista semiestructurada, en la que la niña o el niño escriben sin modelo las palabras que el entrevistador sugiere. Se han usado los protocolos y las plantillas de evaluación de la prueba F/T (Roca Cortés, 1986), material elaborado y validado a partir de la sistematización de las situaciones experimentales usadas por Ferreiro y colaboradores en sus investigaciones.

El paso de la prueba es individual. En la entrevista y el registro, a diferencia de otras pruebas de escritura, se incluyen las vocalizaciones y todos los enunciados verbales que el sujeto emite antes y mientras escribe (tanto si se refieren a la palabra a escribir, a preguntas acerca de la escritura o del acto de escribir, como a comentarios sobre las grafías, sobre sus posibilidades, etc.), el repaso de su propia producción y al final se le pide la interpretación (¿qué has escrito?) y señalamiento (¿dónde lo has escrito?) de su producción escrita.

\section{LAS ESCRITURAS INICIALES DE LOS ALUMNOS CON NECESIDADES EDUCATIVAS ESPECIALES}

\section{Resultados}

Siguiendo la misma descripción de niveles de Teberosky (1989), veamos algunos ejemplos del desempeño en la escritura de los sujetos de la muestra estudiada:

a) escrituras presilábicas:

a.1) escrituras indiferenciadas: ver figura 1, de la sujeto 1 (retraso mental leve, hemiparesia y transtornos del lenguaje), escrito a los 7 años y 11 meses. La escritura de la palabra viernes y su propio nombre es copia (escritura con modelo), el resto es escritura al dictado (sin modelo).

FIGURA 1

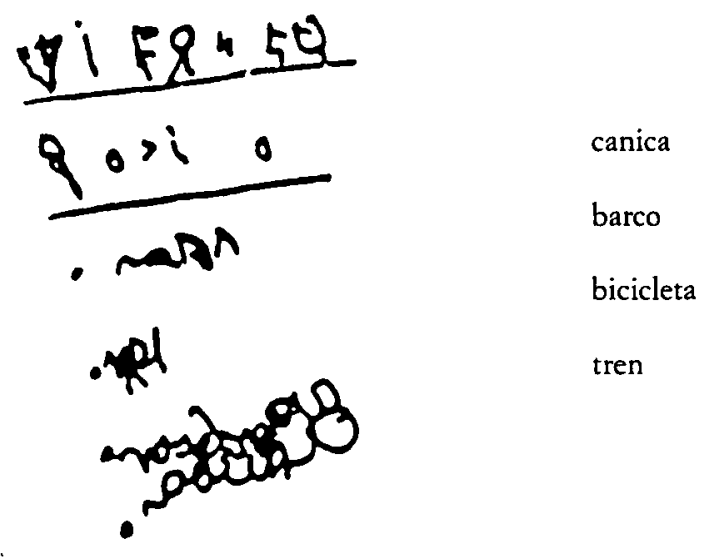


a.2) escrituras diferenciadas, ver figura 2, en la producción del sujeto 5 (retraso límite y transtornos de pronunciación). Observamos la influencia del método fonético usado en el aula común, en la que estaban empezando con las consonantes $\mathrm{p}, \mathrm{l}$.

FIGURA 2

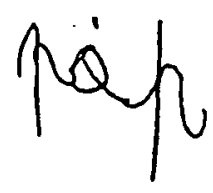

ardilla

león

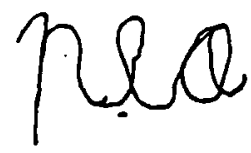

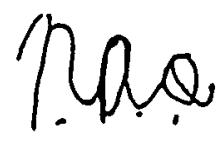

serpiente

pez

Sujeto 5,5 años $y 5$ meses

\section{ESCRITURA DIFERENCIADA}

b) escrituras silábicas: ver figura 3 y 4, del mismo sujeto 3 (retraso mental leve, síndrome de Down, acompañado de transtornos en el lenguaje). La figura 6 corresponde a una producción silábica, pero la correspondencia cualitativa aún no está establecida (no usa las letras con su valor convencional, la mayoría pertenecen a su propio nombre), en la figura 4 ya usa la correspondencia cualitativa según su pronunciación. Estas diferencias de evolución de diferentes aspectos se observan también en muestras amplias de población (obras citadas).

FIGURA 3

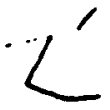

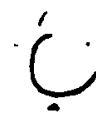

tren

pilota

bicicleta
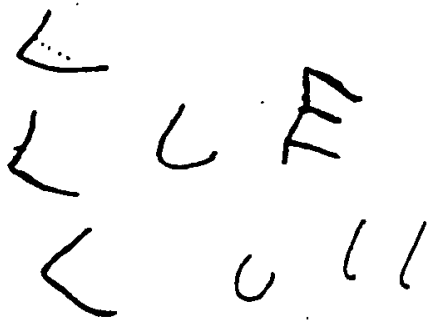


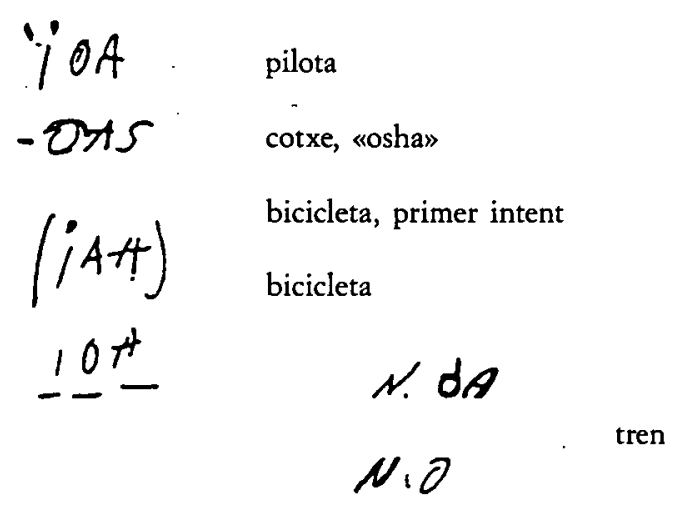

«bi-cie-ta»

Sujeto 3, 9 años.

ESCRITURA SILABICA

CON Valor Sonoro Convencional

La escritura presentada a continuación es la producción obtenida en primera prueba pasada al sujeto 6 (parálisis cerebral), a los 10 años y 8 meses, se realiza con piezas de componer en las que hay signos variados (letras mayúsculas y minúsculas, números, signos aritméticos y otros inventados no convencionales). En la transcripción de su producción se encontrará en minúscula la palabra dictada por la entrevistadora y en mayúscula la producción escrita por el sujeto.

$\begin{array}{llll}\text { ardilla } & \text { AiD } & \text { canica } & \text {.A } \\ \text { león } & . \mathrm{E}< & \text { bicicleta } & \text {.e i B B } \\ \text { serpiente } & \text {.DRe } & \text { barco } & \text {.A C } \\ \text { pez } & . \mathrm{e} & \text { tren } & \text {.3 A L }\end{array}$

los niños juegan con el tren .A R E S $\eta$

Observamos en la escritura un uso mayoritario de la correspondencia silábica, pero no siempre usa las convencionalidades gráficas del valor sonoro (por ejemplo, «ón» de léon). ESCRITURA SILABICA, en las que a veces usa la correspondencia cualitativa (barco, pez, bicicleta, etc.) y en otras no la usa en cada sílaba (león, serpiente, ardilla). En la palabra tren usa el sonido del número tres para representar los sonidos «tre».

Es la primera vez que escribe sin modelo palabras que no son las trabajadas por el método con que se le enseña. Es necesario precisar que llevaba un curso escolar sin apenas trabajar la lectoescritura ya que después de dos cursos de ejercitarse en todas las vocales y tres consonantes $(\mathrm{p}, \mathrm{l}, \mathrm{m})$ se consideraba que apenas había aprendido nada y se debían pues, atenderse otras áreas funcionales.

b.1) escrituras silábico-alfabéticas: se trata de escrituras que se encuentran entre las de tipo b y c. Se observan dos tipos de correspondencia: la silábica y la alfabética. Veamos como ejemplo, en la figura 5, la escritura realizada por la sujeto 1 a los 8 años y 9 meses (aproximadamente un año después de la producción de la figura 1). En algunas ocasiones con la hipótesis silábica usa las consonantes en vez de las vocales: $c a$-ni-ca, bi-ci-cle-ta. La hipótesis alfabética se observa 
en finales de palabra: ca-ni-ca, le-ón, bar-co y en monosílabos: pez, tren. Ambas características se observan también en muestras amplias de población.

FIGURA 5

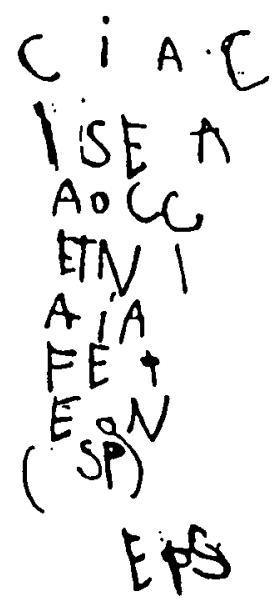

canica

bicicleta

«bisiqueta»

barco, «baco»

tren, «ten»

ardilla, «adiya»

serpiente

«efeiente, fepiente»

león, «eón»

pez, «pes»

Sujeto 1,8 años y 9 meses.

ESCRITURA SILABICO-ALFABETICA

Veamos cómo la sujeto 1 ha realizado algunas palabras. Nos ilustrará qué ocurre con el control de la posición de las letras. Escuchando y siguiendo el proceso de producción de la palabra (y ello es posible por el tipo de prueba que se lleva a cabo) podemos observar cómo se trata de una escritura silábica que en el ensayo de la escritura alfabética se esfuerza solamente en saber cuál es la letra correspondiente que le fata, sin poder controlar aún el lugar exacto que ésta ocupa. El siguiente extracto de protocolo, además de ilustrar el tipo de prueba y su registro, pretende mostrar cómo el conocimiento de los mecanismos que el sujeto usa en su producción escrita puede modificar la evaluación de un resultado final, que en este caso aparece como una inversión de letras (ETN) según el criterio de correspondencia alfabética convencional.

(La transcripción del extracto de protocolo sigue el código siguiente: en la columna de la izquierda se registra la intervención de la entrevistadora, a la derecha la del sujeto, entre comillas aquello que ella dice mientras escribe y en mayúsculas aquello que escribe.)

- Vamos a escribir tren

- Primero me lo dices tú

- Venga, escríbelo

- ¿Cuál es la «n», a ver?

- ¿Cuál es esta que has escrito?

-Tú, ¿cuál buscas ahora?

-Yo te escribo unas cuantas

$-\ll$ Ten»

— «teee» $\mathrm{E}$, «eennn, la «n», ¿cuál es «N»?

- «la de Nono y la de nen»

(nota: nen en catalán, niño en castellano,

Nono, apócope de Antonio) (va a la pizarra $y$ escribe una $P$ ).

$y$ tu escoges la que te parez-

ca que es la «N», ¿vale?

— «la de nen..., no sé cual es»

- «tennn, la " $n$ ". 


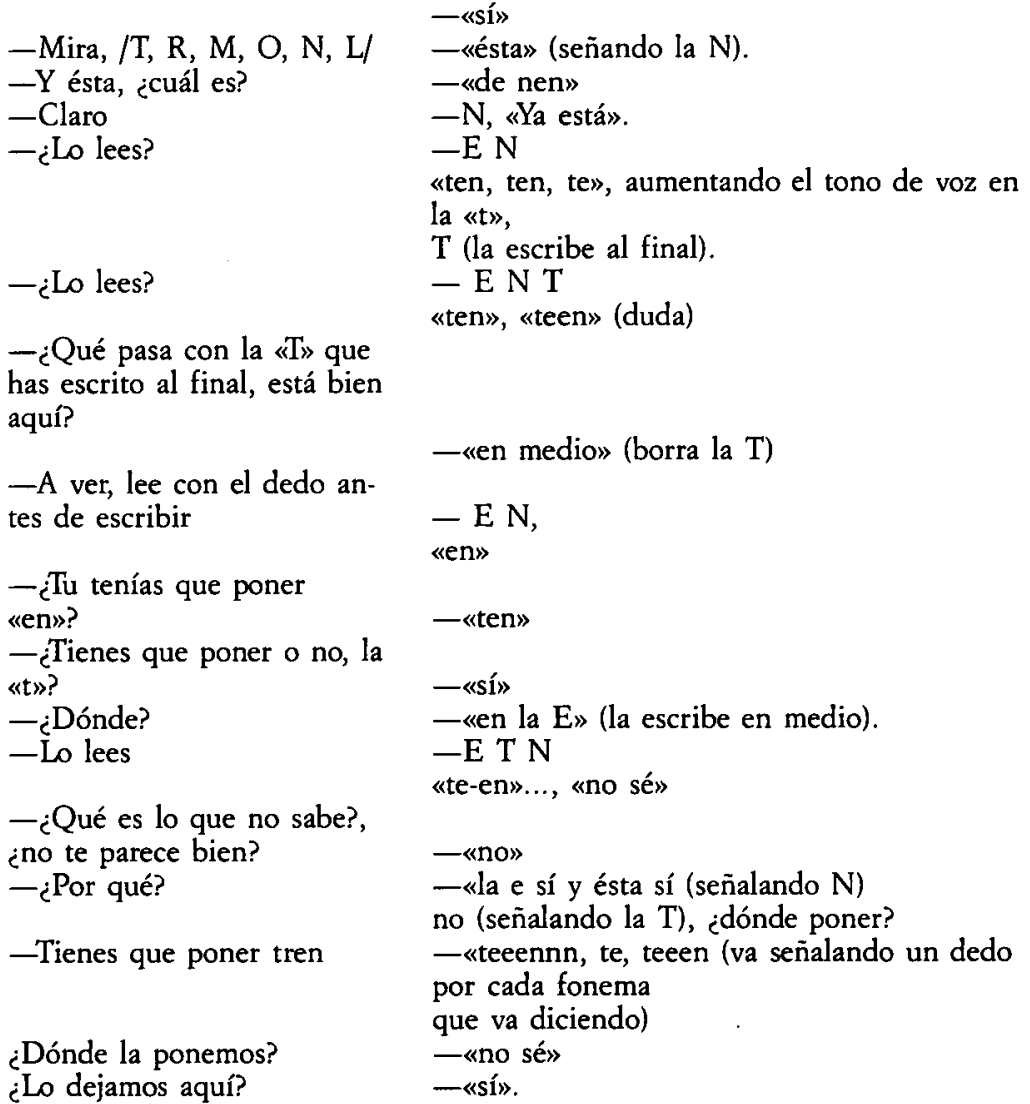

La sujeto 1, en esta ocasión, nos expone claramente sus dudas. Este fenómeno se observa también en las palabras canica, barco y pez. A medida que aumenta el dominio de la correspondencia alfabética, controla paulatinamente la posición de las letras (ver figura 9). Este fenómeno evolutivo también aparece en muestras amplias de población (ob. cit.).

La siguiente producción es del sujeto 7, afectado de parálisis cerebral no puede mover ni manos ni dedos, tampoco habla, y sólo emite sonidos inidentificables, su audición no padece deficiencias. A sus 11 años y 8 meses, es el tercer curso que está en proceso de enseñanza -aprendizaje del lenguaje escrito, y escribe con el procedimiento que llamamos «escriba maquinizada» (con las letras de componer a vista del alumno, la profesional las va colocando- sobre una tabla, según órdenes de éste comunicadas a través de signos no vocálicos). Algunas palabras han sido enunciadas por la profesional más de una vez, ya que puede tardar de 3 a 4 minutos en escribir una sola palabra.

$\begin{array}{lll}\text { pelota } & \text { PELOTA bicicleta } & \text { VISA } \\ \text { coche } & \text { COHKE tren } & \text { TEA } \\ \text { A niñas juegan con el tren } & \text { A NA JEGACONT } & \end{array}$

Se trata de una ESCRITURA casi ALFABETICA

d) escrituras alfabéticas: ver figura 6, escritura de la sujeto 1 a los 10 años y 10 meses ( 3 años y 3 meses después de las escrituras de la figura 1), entre comillas se encuentra la palabra tal cual la pronuncia ella. Y la figura 10, de la sujeto 2 , a los 10 años y 8 meses, escritura con imagen. 
Figura 6

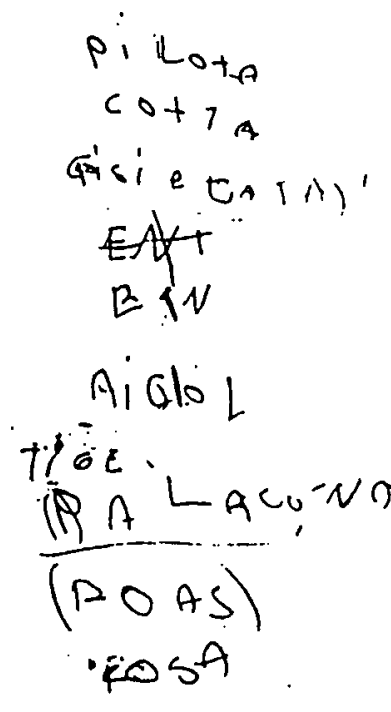

pilota

cotxe, «cotxa»

bicicleta

«bisiteca»

tren, «ten»

esquirol, «astico»

papallona

«palalona»

tigre, «tigue»

Rosa, «Dosa»

Sujeto 1, 10 años y 10 meses

ESCRITURA ALFABETICA

Las siguientes escrituras son producciones ALFABETICAS del sujeto 6 (parálisis cerebral), a los 13 años. Desde la primera escritura sin modelo han transcurrido 2 años y 4 meses. Como se puede comprobar a través del registro de la prueba, aún con sus evidentes dificultades de articulación, usa mecanismos de fonetización (convierte los sonidos «ñ» en «ni», «lle» en «i») y comparaciones fonéticas (mo-ca con mo-ta, de montaña) que transcribimos (en minúsculas y entre comillas) juntamente con su producción escrita (en mayúsculas). Las palabras son dictadas (a la izquierda del registro) de forma continuada («ardilla») o silabeando («ar-di-lla») y esperando a que él escriba después de pronunciar cada una de las silabas (su producción en estas condiciones sigue siendo alfabética pero sensiblemente más convencional). Estos comportamientos también se observan en alumnos de muestras amplias de población.

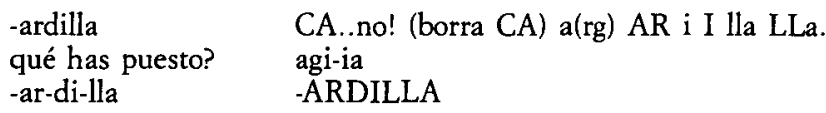

(se dicta silabeando, y esperando a que haya escrito cada sílaba para pasar a la siguiente).

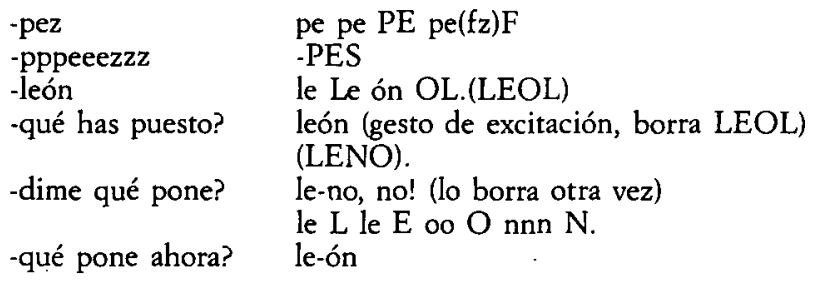

(Las correcciones deben ser incitadas cuando escribe a máquina con una mesa alta ya que le requiere la costosa tarea de incorporar su tronco) 
-montaña

-serpiente -qué has puesto? -mantequilla -mannn-te qui-llla mmm $\mathrm{M}$ mo $\mathrm{O}$ ta mo-ca ia mo ta $\mathrm{TA}$ ni a NI TA.

-MONTANITA ssse SE pi PI pie iiieee LLE te ttt TE. sepiete ma MA que QUE iiIga... ga GA -MANTEQUILLA
-SEPILLETE

-MAQUEIGA

En cuanto a las exigencias de cantidad minima y variedad interna observemos algunos comportamientos que también se dan en alumnos de amplia población:

- en todas las escrituras, sean con o sin correspondencia sonora, observamos que siempre hay más de una grafía. A excepción de la escritura silábica (figura 3) en la que la hipótesis silábica resuelve con firmeza el conflicto que se crea al combinar la aplicación de la correspondencia silábica al monosílabo y la exigencia en cuestión. En otras palabras también monosilábicas (figura 4, 5) la resolución de tal conflicto supone añadir grafías, que pueden usarse con valor sonoro convencional (pez, tren de figura 5) o sin él (tren figura 4).

- en todas las escrituras, sean con o sin correspondencia sonora, no observamos dos grafías consecutivas que sean iguales. A excepción de la palabra barco en la figura 8 , en la que predomina la necesidad de asignar una correspondencia alfabética a «co».

Veamos dos ejemplos de cuando esta exigencia se convierte en conflicto: la palabra bicicleta en niveles de escritura silábica y silábico-alfabética podría suponer la escritura de dos íes seguidas. El sujeto 3 actúa cambiando la grafía y por la a, perdiendo así el valor sonoro «ci», pero a pesar de ello le quedan dos letras a seguidas con lo cual necesita volver a escribir la palabra cambiando esta vez la i de «ci» por la o (perdiendo el valor sonoro convencional ante el conflicto). En cambio la sujeto 1, en la figura 5, ante el mismo conflicto sustituye la segunda i de «ci» por la s (en catalán «ci» se pronuncia «si»), con lo cual no pierde el valor sonoro convencional.

\section{CONCLUSIONES}

En los 8 sujetos estudiados, los resultados nos muestran que:

1. Desde el momento en que ha sido pasada la primera prueba, todas las producciones escritas de todos los sujetos de la muestra, pueden ser entendidas e interpretadas desde la descripción de las hipótesis y niveles del proceso de adquisición del sistema alfabético notacional, realizado por Ferreiro y colaboradores $(1979,1982,1987$, etc.) en muestras amplias de población. Así pues, podemos concluir que ello es así sea cual sea la edad del sujeto, la discapacitación o alteración y las condiciones de escolarización.

Las exigencias que el niño desarrolla a lo largo de su aprendizaje y sus formas de resolución, observadas en muestras amplias de población (Ferreiro y Teberosky, 1979; Ferreiro y Gómez-Palacio, 1982), han sido también constatadas en estos sujetos.

Mecanismos de fonetización, comparaciones fonéticas y el control de la posición de las letras son comportamientos que también son observados en muestras amplias de población.

Todos estos aspectos han sido ilustrados con los ejemplos comentados anteriormente.

2. En todos los sujetos el orden de sucesión de los niveles que presentan sus 
producciones escritas corresponden al orden secuencial que desarrollan los sujetos de muestras amplias de población. Y ello es así sean cuales fueran su discapacidad o alteración y su edad.

En la tabla 2 y 3 se muestran los sucesivos niveles por los que han evolucionado las producciones escritas de los sujetos de la muestra, en los plazos de tiempo que duró el estudio. Los sujetos se encuentran agrupados por tipos de discapacidades (tabla 2) y por tipos de alteraciones del lenguaje (tabla 3). Observamos cómo a partir del nivel apreciado en la primera prueba se han ido sucediendo las etapas de la forma prevista en muestras amplias de población. Observamos que algunos sujetos no han llegado aún a la correspondencia convencional en el momento final del estudio, lo cual no implica que no sigan evolucionando.

\section{TABLA II}

Evolución de la escritura según el tipo de discapacidad

\section{Tipo de discapacidad o alteración}

Sujetos/Niveles

retraso mental leve

$\begin{array}{cccccc} & \text { Indif. } & \text { Difer. } & \text { Siláb. } & \text { Sil.alfab. } & \text { Alfabet } \\ 1 \ldots \ldots \ldots \ldots . & + & + & + & + & + \\ 2 \ldots \ldots \ldots \ldots . & & + & + & + & + \\ 3 \ldots \ldots \ldots \ldots \ldots & + & + & + & + & \end{array}$

retraso mental limite

$\begin{array}{cccccc} & \text { Indif. } & \text { Difer. } & \text { Siláb. } & \text { Sil.alfab. } & \text { Alfabet. } \\ 4 \ldots \ldots \ldots \ldots \ldots & + & + & + & + & + \\ 5 \ldots \ldots \ldots \ldots & + & + & + & + & +\end{array}$

parálisis cerebral

$\begin{array}{lccccc} & \text { Indif. } & \text { Difer. } & \text { Siláb. } & \text { Sil.-alfab. } & \text { Alfabet. } \\ 6 \ldots \ldots \ldots \ldots & & & + & + & + \\ 7 \ldots \ldots \ldots \ldots . & & + & + & + & +\end{array}$

Alteración de la escolaridad

$\begin{array}{cccccc} & \text { Indif. } & \text { Difer. } & \text { Siláb. } & \text { Sil.-alfab. } & \text { Alfabet. } \\ 8 \ldots \ldots \ldots \ldots . . . . . & + & + & + & + & \end{array}$

\section{Algunas peculiaridades}

En comparación cón el proceso de adquisición en alumnos sin discapacidades, se apuntan algunas pecualiaridades, por otro lado previsibles:

A. Coincidiendo con la experiencia de psicólogos y otros profesionales observamos que el tiempo empleado por este alumnado en el aprendizaje ha sido 
TABLA III

Evolución de la escritura seguin los transtomos del lenguaje.

\section{Transtomos del lenguaje \\ Sujetos/Niveles}

Presentan transtomos:

Transtomos en la pronunciación y la estructuración

$\begin{array}{lccccc} & \text { Indif. } & \text { Difer. } & \text { Siláb. } & \text { Sil.-alfab. } & \text { Alfabet. } \\ 1 \ldots \ldots \ldots \ldots . & + & + & + & + & + \\ 3 \ldots \ldots \ldots \ldots . & + & + & + & + & \\ 6 \ldots \ldots \ldots \ldots . & & & + & + & + \\ 7 \ldots \ldots \ldots \ldots . & & + & + & + & +\end{array}$

Transtomos solamente en la pronunciación

$\begin{array}{cccccc} & \text { Indif. } & \text { Difer. } & \text { Siláb. } & \text { Sil.-alfab. } & \text { Alfabet. } \\ 4 \ldots \ldots \ldots \ldots & + & + & + & + & + \\ 2 \ldots \ldots \ldots \ldots \ldots & & + & + & + & +\end{array}$

No presenta transtomos:

$\begin{array}{cccccc} & \text { Indif. } & \text { Difer. } & \text { Siláb. } & \text { Sil.-alfab. } & \text { Alfabet. } \\ 5 \ldots \ldots \ldots \ldots . & + & + & + & + & + \\ 8 \ldots \ldots \ldots \ldots . & + & + & + & + & \end{array}$

bastante más largo que el que generalmente emplean alumnos y alumnas sin dificultades. Es de notar que aquellos alumnos con retraso mental límite han empleado menos tiempo que los alumnos con retraso mental leve (ver edades en tabla I). Las imposibilidades motrices, las deficiencias en la abstracción, etc. son motivo de este enlentecimiento. No sabemos cómo incide este factor tiempo en la cantidad y calidad del proceso y en su resultado final.

B. En otro orden de cosas, cabría decir que la adquisición de la correspondencia cualitativa ha sido también posterior, en el tiempo, a la cuantitativa. Pero este desfase parece haber sido mayor en sujetos con retraso mental leve acompañado de alteraciones linguísticas en la pronunciación y la estructuración (Sujetos. 1 y 3).

Los resultados aportados hasta el momento suponen una primera aproximación a la aplicación de esta perspectiva a alumnos con discapacidades. Tal vez un estudio a la vez más específico y amplio permitiría la identificación (si se dan) de algunos comportamientos pecualiares y/o exclusivos favorecidos por un perfil diferenciado de combinación de capacidades y discapacidades de los sujetos. Este ha sido el objetivo de Bellés (1989) en su estudio de la adquisición del sistema notacional alfabético en niños sordos.

\section{Las discapacidades y el proceso de adquisición}

Por la evolución y los resultados de los sujetos del estudio, podemos esbozar qué relaciones se dan (tal como las entenderíamos desde este enfoque) entre características de las discapacidades y el proceso de adquisición de la escritura: 
1. Las deficiencias motrices interfieren en la plasmación gráfica del producto escrito y en la cantidad de posibilidades de ejecución, pero no impiden el aprendizaje del sistema de escritura. Debidamente compensadas por letras de componer, máquinas de escribir o por escribas maquinizadas, estos alumnos han podido realizar producciones de niveles más elevados que trabajando con su propia mano, o si no podían mover sus manos han tenido ocasión de aprender a escribir (Sujetos 1, 6 y 7).

2. en cuanto a las alteraciones lingüísticas.

a) Las dificultades de pronunciación (entendiendo los déficits de articulación) no han sido un obstáculo para llegar al uso del sistema alfabético de correspondencia sonora, en su aspecto de correspondencia cuantitativa. Tampoco ha sido un grave obstáculo para la consecución de la correspondencia cualitativa, es decir, atribuir el valor sonoro convencional a la letra según el fonema pronunciado, a pesar de necesitar más tiempo aquellos sujetos que presentaban ambas dificultades, la de pronunciación y las de categorización (con similares resultados se ha encontrado Bellés, en su estudio con niños sordos). Se ha alcanzado paulatinamente la correspondencia alfabética, a medida que el análisis sonoro del enunciado ha sido más fino. Y ello también ha ocurrido en el caso del sujeto 7, que no habla por imposibilidad motriz, aunque oye perfectamente. La dificultad real encontrada es que escriben tal cual ellos pronuncian (en el caso del sujeto 7 suponemos que es tal como rememora).

Así pues, el problema radica en la comunicabilidad de sus escritos (más que en no conocer la correspondencia alfabética sonora convencional), en tanto que las palabras tal cual están escritas («arilla» por ardilla, «reinte» por serpiente, etc.) no tienen significados socialmente compartidos.

En este sentido hemos observado en ellos un esfuerzo consciente y adicional en pronunciar según el uso socialmente correcto, o ensayar variaciones en la pronunciación mientras están escribiendo (también cuando leen; no obstante, este aspecto merece consideración aparte). Pero este comportamiento solamente se ha observado cuando el sujeto está usando la hipótesis alfábética (ejemplo de la segunda escritura del sujeto 6,). Ello puede ser explicado desde dos vertientes: desde la interpretación de la evolución de los niveles, en tanto que en etapas anteriores no se ha establecido aún una correspondencia sonora alfabética que afecta a la precisión fonética; y desde la interacción social institucional, el adulto y los demás compañeros informan de la norma: hay una manera de pronunciar ún significado y no otra.

Debido a la toma de conciencia del aspecto fonológico que supone este nivel, podemos pensar que se trata de un momento especialmente idóneo para los tratamientos foniátricos.

b) Las dificultades de categorización y estructuración del lenguaje (concordancias incorrectas, omisión de nexos, etc.) se ven siempre reflejadas en sus composiciones escritas espontáneas $\mathrm{y}$, por tanto, pueden ser más o menos comunicables.

c) En algunos alumnos creemos se han dado, con bastante claridad, las condiciones de pobreza en su experiencia social interactiva con el lenguaje escrito (descrito por varios autores, entre ellos G. Wells, 1988): al sujeto 6 (parálisis cerebral), se le había enseñado, en tres años, solamente las pocas palabras que tienen las vocales y las consonantes $\mathrm{p}, \mathrm{l}, \mathrm{m}, \mathrm{n}$; al sujeto 8 no le suponemos contacto activo alguno, por pertenecer a una cultura tradicionalmente oral, vivir en el campo y no haber estado nunca escolarizado. En ellos hemos podido comprobar la im- 
portancia de este factor en el desempeño en la escritura y las peculiaridades de su producción, en tanto que la evolución del aprendizaje de estos alumnos ha sido muy rápida desde el momento que inciaron el trabajo con nosotras y por otra parte no presentan deficiencias mentales.

\section{IMPLICACIONES}

Con la cautela necesaria derivada de las características metodológicas de este estudio, los datos empíricos aportados con sus conclusiones abren preguntas y suscitan revisiones de conceptos y prácticas de diagnóstico y enseñanza especial a la vez que propuestas alternativas, que intentaremos esbozar aquí.

\section{Para la evaluación: ¿error o avance?}

La consideración del aprendizaje de la escritura como un proceso de construcción representacional, y de las manifestaciones escritas por las que discurre, nos obliga a una revisión del concepto de «error» de escritura. Concepto profusamente utilizado en múltiples tests de lectoescritura y en programas de reeducación de dificultades en la escritura, tiene su origen en un enfoque asociacionista del aprendizaje que aplicado a la lectoescritura considera el aprendizaje de ésta como un acceso directo al código de transcripción.

Si se estiman las producciones escritas desde una óptica genética, es decir, de proceso paulatino de aprendizaje, en el que las correspondencias sonido-grafía convencionales son el punto final de un camino y no su punto de partida, el concepto «error» pierde su significado de distorsión o alteración. Así pues, y desde este enfoque genético, al referirnos a estas escrituras con «errores» (en el sentido usado por la mayoría de autores como errores de correspondencia grafíasonido - «ortografía natural»-, y no errores relativos a la ortografía en sí mis$\mathrm{ma}$ ), deberíamos hablar de escrituras significativas no convencionales, producidas con una lógica diferente a la del producto final esperado (sin olvidar que a ello se tiende), que poseen un esquema de conocimiento peculiar, no arbitrario.

Veamos algunos ejemplos de las diferentes interpretaciones de una mismá escritura:

- las «omisiones» de letras (faltan una o algunas letras en una palabra) pueden ser resultado de un modo de construcción silábico alfabético (por otra parte, una de las fases más avanzadas del proceso),

- las «inversiones» de letras (alteración del orden de las letras en una palabra) de una sílaba (como la del sujeto 1 en la figura 5 y 6: pez, tren) pueden ser entendidas como resultados de un proceso de análisis de la palabra sin consideración del orden de correspondencia, fruto de una inseguridad inicial (argumentado y detallado en el comentario de sus producciones),

- una escritura con «omisiones» e «inversiones» (figura 5, 6: canica, barco) puede ser una escritura silábica en transición inicial hacia la correspondencia alfabética (idem anterior).

- las «sustituciones» (en lugar de la letra correspondiente se usa otra que no es la correcta) pueden tratarse de correspondencias alfabéticas sin valor sonoro convencional o estratégias necesarias para solucionar los conflictos de cantidad mínima y variedad interna (detallado y explicado en el apartado correspondiente), 
- las «uniones» y «separaciones» arbitrarias se segmentos de palabras o palabras enteras (aspecto no tratado en este artículo) pueden ser entendidas como una etapa en el proceso de adquisición de este aspecto arbitrario estudiado por Ferreiro y colaboradores $(1979,1982)$.

Se consideraría, pues, que no se trata de «errores» sino de un paso cognitivo necesario hacia la consecución de la meta de apropiación de un objeto de conocimiento (el sistema alfabético y la ortografía), que deben reconocerse como tales y por tanto no reprenderlos o eliminarlos para ser sutituidos sino permitirlo y sobre todo usarlos como base para estimular los avances propios del proceso.

Acaso debería considerarse «error en la escritura» una vez que el desempeño del alumno llegue al nivel en que aplique a cada uno de los sonidos de la palabra la correspondencia alfabética convencional y siendo así, de forma permanente incurra posteriormente en estos errores o no evolucione (aun así deberíamos conocer el proceso de aprendizaje de la ortografía, en la cual los alumnos también apliçan sus hipótesis antes de llegar a las convencionales).

Se hace, pues, necesario que en el diagnóstico y evaluación de las «dificultades o transtornos en la escritura", además de valorar el rendimiento escolar en escritura (comparar la escritura del alumno con la esperada por la escuela), se introduzca un estudio genético del desempeño del sujeto en la escritura. Ello implicaría no solamente la consideración de la génesis sino también del tipo de prueba que se usa en este enfoque.

Usar esta evaluación desde edades tempranas permitiría detectar precozmente a aquellos alumnos de ritmo más lento sin tener que esperar a que más adelante sean considerados como fracasados (y además sin que se les pueda reconocer sus avances, Roca Cortés, Grau, Adroher, Rovira, Abril, Nogués y Mateu, 1990).

Esta nueva propuesta de cambio conceptual supondría cambios también en la enseñanza, en sus objetivos, y sobre todo cambios importantes en la relación alumno-maestro. Si su escritura representa un «avance» y no un «error», la percepción de la capacidad del alumno tiende a ser más positiva y consecuentemente aumentan las expectativas de evolución y mejora tanto para el alumnado mismo como para profesionales y padres. De todos es sabido que el signo y calidad de las expectativas tiene un elevado grado de influencia en el rendimiento del alumnado, incluso en alumnos con dificultades.

Estos aspectos son especialmente relevantes en educación especial, ya que las reales dificultades que el alumnado presenta y el lento ritmo de su desarrollo provocan por sí mismas tendencias al pesimismo. La consideración de avance en vez de error puede balancear esta tendencia y beneficiar en último término al mismo alumnado (así se ha constatado en nuestra experiencia de enseñanza, observando en aquellos alumnos que se les había trabajado antes desde otros enfoques una notable explosión de su motivación).

Por otra parte y en otro orden de cosas, puede resultar fácil que la explicación de algunos fracasos de los alumnos con dificultades sea únicamente atribuidos a sus discapacidades cuando también podrían estar relacionados con las prácticas y las concepciones de la enseñanza.

\section{Las «habilidades previas» en educación especial, ¿para qué?}

Si los problemas motrices (presentes desde el nacimiento) que afectan al movimiento de las manos no constituyen un obstáculo para aprender a escribir, podría pensarse que no constituye un prerrequisito para el aprendizaje de éste. Es 
evidente que tal impedimento sí afecta al uso autónomo de la escritura como instrumento de comunicación, a la cantidad de posibilidades de ejercitación de la escritura y a las posibilidades de desempeño en la más o menos bella y clara ejecución de los caracteres gráficos. Pero los datos reseñados en el estudio (y a pesar de sus limitaciones) apuntan a sostener que las habilidades motrices no constituyen un requisito para el aprendizaje del sistema notacional. Si para los especialistas en parálisis cerebral ello ha sido siempre bastante evidente, para muchos enseñantes de educación especial en escuelas ordinarias o con alumnos sin esta deficiencia específica no ha resultado así. En la práctica reeducativa las incoordinaciones motrices han sido y son aún hoy motivo para retrasar o ralentizar la enseñanza-aprendizaje de la lectoescritura.

Este es un aspecto importante también a tener en cuenta cuando se secuencian y seleccionan los contenidos a trabajar en un programa adaptado, cuando en algunos programas de reeducación se ocupan muchas horas en elaborar materiales o realizar ejercicios de tipo motriz con el objetivo de estimular la madurez para la escritura.

Otros estudios apuntan también hacia tal afirmación mostrando la débil contribución de este factor en las habilidades para la lectoescritura (Jiménez y Artiles, 1990) o su nula capacidad de discriminación en las dificultades del rendimiento escritor (Tolchinsky y Landsman, 1982).

La evolución de nuestros alumnos y alumnas nos sugiere revisar el criterio de que una correcta pronunciación del lenguaje es un prerrequisito para el aprendizaje del sistema alfabético. No hemos esperado a que nuestro alumnado pronunciara y hablara más o menos correctamente para iniciar la enseñanza del lenguaje escrito. Aun con sus evidentes dificultades de pronunciación han ido aprendiendo el sistema alfabético de correspondencia.

Las razones que nos han llevado a desestimar el trabajo con las consideradas habilidades previas han sido: las tareas que suelen usarse para estimular tales habilidades no ejercitan la lectura y la escritura propiamente dicha y consecuentemente se resta tiempo al aprendizaje de éste (evidencia puesta a la luz por otros profesionales, Huerta y Matamala, entre otros); la constatación de que éste aprendizaje es fundamentalmente un aprendizaje cognitivo y donde lo perceptivomotriz no es un dominio aislado sino relacionado con lo cognitivo, también para los alumnos con deficiencias o alteraciones en su escolaridad.

En este sentido y también para los alumnos con deficiencias o alteraciones en el rendimiento escolar es válido el posicionamiento de la perspectiva o piscogenética al respecto. Teberosky (1987) lo formula así:

«esta modalidad de trabajo nos permite superar el dilema de los «prerrequisitos»... en el sentido de habilidades mínimas-pero necesarias para llegar a resolver una tarea... En efecto, si sabemos que los niños pueden realizar una tarea según sus posibilidades, cada cual según su nivel, no es necesaria la preparación previa para pasar de una tarea a otra.»

Habitualmente las discapacidades justifican el aumento del tiempo dedicado a los prerrequisitos y por tanto retrasan el inicio del aprendizaje de la lengua escrita propiamente dicha. La estimulación (o sustitución) de estos aspectos perceptivo-motrices en nuestro alumnado ha sido necesaria, en algunos casos, no como prerrequisito del aprendizaje de la escritura sino como desarrollo de este aspecto en general (también para la escritura pero entre otras múltiples tareas escolares). 
No obstante, es evidente que el lenguaje va más allá de la pronunciación. El lenguaje, en general, independientemente del canal por el cual se emite o recibe pasa a ser aquello a representar sin lo cual aún adquirido el sistema, la escritura no sería posible.

Ello implica, a nuestro entender, reexaminar algunas calificaciones de escrituras no convencionales (como por ejemplo la escritura de la sujeto 2 , figura 7 , o las realizadas por la sujeto 1 en textos largos) consideradas como transtornos o dificultades en la escritura, cuando en realidad (y se hace evidente cuando llegan a la correspondencia alfabética sonido-grafía) suele tratarse no de dificultades en la adquisición del sistema, sino de alteraciones en el lenguaje propiamente dicho, es decir, en la competencia lingüística. Esta distinción debe realizarla tanto un diagnóstico como los objetivos y actividades de la enseñanza especial. Si las dificultades se centran en el lenguaje, las actividades deben ir dirigidas hacia la mejora de esta competencia, sea a través de la conversación o composición (oral) o a través del lenguaje escrito.

FigURA 7

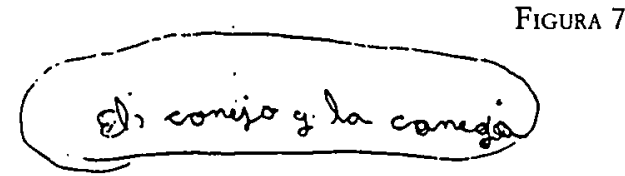

Imagen: dos conejos en un campo de hierba.

Uno de ellos tiene una zanahoria

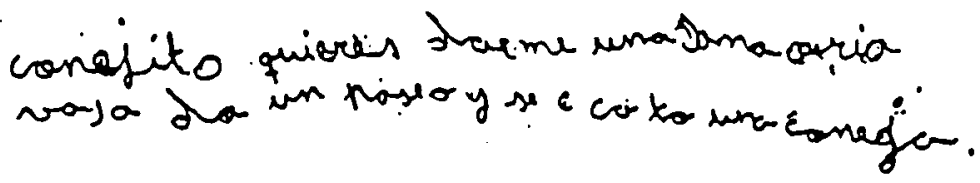

Sujeto 2, 10 años y 8 meses.

\section{ESCRITURA ALFABETICA (Discuriva)}

En cuanto al aspecto social, y en relación al diagnóstico, cabría solamente proponer la inclusión de un estudio (o como mínimo una aproximación valorativa) de la calidad de la interacción del alumno con textos escritos y con usuarios de ellos, en sus contextos sociales de convivencia (escuela, familia, entorno, etc.).

En cuanto a la enseñanza, y después de lo expuesto, es necesario concluir que aplazando el aprendizaje del lenguaje escrito se corre el gran riesgo de empobrecer el contacto activo del alumno con el conocimiento y específicamente en el caso de los alumnos discapacitados con la lentitud que suele acompañarles ello puede representar un peligro aún mayor. Con estas medidas se consiguen efectos contrarios a los pretendidos: provocar «no avances» en su desarrollo.

Si nos permitimos considerar que los alumnos discapacitados también pueden trabajar a su nivel (hemos visto que siguen los niveles del proceso de adquisición), también para ellos el mejor trabajo «previo» para un mejor aprendizaje de la lectoescritura es el contacto (Ferreiro, 1982) con textos escritos, con lectores y escritores que les permitan participar activamente (y no solamente mecánicamente) en tales funciones. 
Las adaptaciones pedagógicas en alumnado con necesidades educativas especiales

Si tales investigaciones permiten a los y las profesionales de la enseñanza saber las hipótesis y los conocimientos que subyacen tras el desempeño del alumnado, y también cuáles son las próximas etapas en el desarrollo de la escritura, se podrá comprender e interpretar (con sólida base) aquello que ya saben y pueden llegar a saber. Permitiendo así una adecuación más específica de las tareas pedagógicas y de las decisiones escolares. Sería, pues, presumible pensar en la obtención de resultados más óptimos en la enseñanza de la escritura. Los criterios que se desarrollarán partirán del análisis de lo que el niño construye en interacción con el objeto de aprendizaje y no de un análisis analógico del producto a enseñar.

Si ello ya resulta sugerente en alumnos sin dificultades de aprendizaje, es especialmente interesante en aquellos que presentan necesidades educativas especiales. Estos conocimientos son aplicables a decisiones profesionales como la secuenciación de tareas y materiales, la selección de textos, la promoción de nivel o no de un alumno, que a nuestro entender pueden modificar bastante el panorama la enseñanza de los alumnos con necesidades educativas especiales (tanto en aula de educación especial de una escuela común como en una escuela de educación especial), ya que son decisiones que en nuestro ámbito se manejan con frecuencia y de forma consciente, con la intención de facilitar el aprendizaje o reforzarlo.

Aquí solamente abordaremos una de estas decisiones: la adaptación del currículum ordinario a las posibilidades y necesidades especiales. Además del currículum ordinario y el método como guía de los pasos para su enseñanza, también podemos usar el conocimiento de sus posibilidades cognitivas respecto a la escritura en concreto (no respecto a sus habilidades intelectuales generales) siempre que nos permitamos intepretar sus lecturas y escrituras no convencionales no como «error» sino como una escritura significativa no convencional y evolutiva. De este modo no solamente individualizaríamos (pasar a trabajar solo), o parcializaríamos su tarea en subtareas (en las que a menudo la dificultad cognitiva de la tarea es la misma pero con menos cantidad de contenido y más tiempo para realizarlo), sino que se podría adecuar, en el sentido de ensamblar sus conocimientos previos y su nivel cognitivo con los nuevos conocimientos a través de tareas adecuadas a sus posibilidades para que, situándose en tal proximidad, pueda avanzar.

Un ejemplo de inadecuación: la tarea de completar palabras en que falta una letra o escribir los nombres de las imágenes (en ambos casos aunque sólo sean las palabras ya trabajadas o las más «simples» por contener sólo dos-tres consonantes), en la que se espera que la respuesta correcta sea la escritura de todas y cada una de las letras de la palabra, aplicada a un alumno que se éncuentra en un nivel de escritura presilábica o silábica.

Si las actividades que se usan para enseñar a leer y escribir suponen una respuesta del alumno que implica unos esquemas de conocimiento que éste aún no posee y en consecuencia no puede llegar a producir la escritura esperada (de nivel alfabético), nos encontraremos con un trabajo poco fructífero y con repercusiones negativas para el alumno (desmotivación, sensación continua de fracaso, actitudes de evitación o rechazo hacia el lenguaje escrito, disminución de la autoestima, etc.). Como ejemplo: Probablemente el alumno deberá borrar varias veces su escritura, acabará copiándolo de algún compañero o de la misma maestra o no realizará la tarea y «molestará». 
La parcialización de las tareas tiene como consecuencia el retraso en la aparición de nuevos estímulos (inputs) añadiendo más tareas entre uno y otro. Un ejemplo habitual que aparece en los materiales del mercado es la ampliación del número de tareas entre la presentación de una letra y la siguiente, o entre la presentación de frase y frase. Una vez más a través de la dosificación de estímulos se enlentece la estimulación. Y a menudo con ello suele trabajarse por debajo de las posibilidades del alumno. En todo caso con esta medida, una vez más, se merman las posibilidades de interacción con el lenguaje escrito.

Desde la perspectiva del enfoque presentando la adaptación de la enseñan$z a$ de la escritura a los alumnos con necesidades educativas especiales no trataría tanto de reducir la densidad de los estímulos como de ajustar y variar las actividades y exigencias a la zona de desarrollo próximo del alumno, que podremos conocer a través del nivel de desempeño en el que se encuentra.

Es decir, una perspectiva evaluativa que permita proponer una enseñanza que vaya más allá de lo que el niño ya sabe. E insistimos una vez más: aunque también siendo un alumnado con discapacidades o alteraciones en su escolaridad o al contrario, con más razón a causa de ello.

Teniendo en cuenta este aspecto y los otros ya mencionados en este artículo, las alumnas y alumnos de educación especial, además de las limitaciones que comportan sus discapacidades pueden encontrarse en una situación de pobreza lingüística (Clark, 1983) durante bastantes años. Condición que debemos recordar no corresponde en absoluto a sus necesidades, ni tan siquiera a nuestras intenciones.

\section{Referencias}

Belles, R. M. (1989). «Producción e interpretación de textos escritos por niños sordos pequeños integrados en escuelas ordinarias». Convocatoria de Proyectos de Investigación Educativa de 1985. CIDE. Barcelona, marzo, 1989.

Cenvera, M.; Toro, J. (1980). Test de Análisis de la Lectura y la Escritura. Madrid: Pablo del Río Editor.

CLARK, M. M. (1983). «Orientaciones actuales en la enseñanza y aprendizaje de la lectura», a Schaffer, H. R. (comp.), Nuevas perspectivas en Psicología del descrrollo de la lengua inglesa, Monografía de Infancia y Aprendizaje, $n .^{\circ} 3$, págs. 185-201, Madrid.

Ferreiro, E., y Teberosky, A. (1979). Los sistemas de escritura en el desarrollo del niño, México, Siglo XXI.

Ferreiro, Gómez-Palacio y colaboradores (1982). Análisis de las perturbaciones en el proceso escolar de la lectura y la escritura, México: Dirección General de Educación Especial.

Huerta, E.; Matamala, A. (1983). Programa de reeducación para dificultades en la escritura. Manual para el reeducador, maestro y padres. Madrid: Aprendizaje Visor.

JimÉnez, J., y ArTiles. C. (1990). Factores predictivos del éxito en el aprendizaje de la lectoescritura, en Infancia y Aprendizaje, $n .^{\circ} 49$, págs. 21-38.

Molinas, S. Enseñanza y aprendizaje de la lectura. Madrid: CEPE.

Roca Cortés, N.; Simo Águade, R.; Solsona Roca, R.; Rabasa del Campo, González Garcia (1991). Ensenyament-aprenentatge de l'escriptura a nens i nenes amb necessitats educatives especials. I.C.E. (Institut de Ciències de l'Educació) de la Universidad de Barcelona.

Roca Cortés, N. (1990). Avaluació sistemática de l'adquisició de l'escriptura i la lectura. Barcelona 1990. Mecanografiado no publicado.

Roca Cortés, N.; Grau, R.; Adroher, O.; Abril, T.; Rovira, C.; Mateu, A.; Nogues, D. (1990). «Lectura, escriptura i educació especial a l'escola ordinària. Estudi comparatiu». Informe de Investigación del Seminari Permanent sobre Intervenció Psicopedagògica i Llenguatge Escrit, de l'Institut de Ciències de l'Educació (ICE) de la Universidad de Barcelona.

Teberosky, A. (1982). «Construcción de las escrituras a través de la interacción grupal», en $\mathrm{Fe}$ rreiro y Gómez-Palacio (comp.): Nuevas perspectivas sobre los procesos de lectura y escritura», México: Siglo XXI. 
Teberosky, A. (1984). «La intervención pedagógica y la comprensión de la lengua escrita» a Lectura y Vida, Buenos Aires. Diciembre.

Teberosky, A. (1987). Psicopedagogia de la llengua escrita, IME (Institut Municipal d'Educació de Barcelona).

Tererosky, A.; Cardoso, B.; Donat, A.; Cuquerella, M.; Barrios, O.; Vinardell, R., y Pérez, R. (1989). Psicopedagogia de la llengua escrita. Documents de classe, IME (Institut Municipal d'Educació de Barcelona).

Teberosky, A. (1989). «Didàctica e intervención en el aula del lenguaje escrito», En La Lectura, págs. 101-106. V Simposio de Escuela de Logopedia y Psicología del Lenguaje.

TolchinsKy, L., y LeVIN, I. (1982). «El desarrollo de la escritura en niños israelís preescolares», en Ferreiro y Gómez-Palacio (comp.): Nuevas perspectivas sobre los procesos de lectura y escritura», México: Siglo XXI.

TolcHINSKY, L. (1989). «Lengua, escritura y conocimiento lingüístico», ponencia presentada al XIV Seminari «Llengues i Educació», Sitges.

$\mathrm{W}_{\mathrm{ELLS}}, \mathrm{G}$. (1988). Aprender a leer y escribir, Barcelona: Laia.

\section{Desarrollo del aprendizaje de la escritura en alumnos y alumnas discapacitados Neus Roca Cortés $C L \& E, 16$, pp. $61-82$}

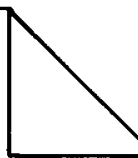

Resumen: Exponemos en este artículo cómo las producciones escritas de alumnos con diversas discapacidades (parálisis cerebral, deficiencia mental, retraso mental límite, etc.) o alteraciones de su escolaridad también pueden ser entendidas desde la descripción de niveles realizada en alumnos de amplia población. Siendo ello así, se nos permite comprender escrituras no convencionales hasta el momento tratadas como síntoma de transtorno o dificultad en la escritura y, por tanto, revisar y redefinir algunos criterios psicopedagógicos al uso en educación especial. Los «errores» de la escritura pueden ser avances (construcciones evolutivas). El trabajo previo con habilidades «prerrequisitas» no ayudaría al desarrollo de la escritura sino que lo retrasaría. Se matizan algunos efectos de los transtornos de lenguaje en el aprendizaje de la escritura. De individualizar y parcializar como estrategias de adaptación se propone la adecuación cognitiva. Se alerta sobre el empobrecimiento excesivo que algunas prácticas derivadas de tales criterios provocan en los entornos de enseñanza de este tipo de alumnado, consiguiendo efectos contrarios a los propuestos. Se propone una evaluación que al permitir conocer lo que ya saben se pueda plantear una enseñanza que vaya más allá de lo que ya saben, también para los alumnos y alumnas discapacitados.

Datos sobre la autora: La autora trabaja en el Equipo de Asesoramiento Psicopedagógico de la comarca del Garraf, Barcelona.

Dirección: Equip d'Assessorament Psicopedagògic (E.A.P.) de la comarca del Garraf (Barcelona, Cataluña), Placa Soler i Carbonell, 29, 3. ${ }^{\mathrm{a}}$ planta, despatx n. ${ }^{\circ} 5.08800$ Vilanova i la Geltrú. Universidad de Barcelona. Facultad de Psicología.

(C) De todos los artículos deberá solicitarse por escrito autorización de CL\&E y de los autores para el uso en forma de facsímil, fotocopia o cualquier otro medio de reproducción impresa. CL\&E se reserva el derecho de interponer las acciones legales necesarias en aquellos casos en que se contravenga la ley de derechos de autor. 